\title{
A case of endometrial cancer presenting with malignant spinal cord compression
}

\author{
Shigeki Taga ${ }^{*}$, Mari Sawada, Aya Nagai, Dan Yamamoto, Ryoji Hayase \\ Department of Obstetrics and Gynecology, National Hospital Organization Fukuyama Medical Center, Fukuyama, Japan \\ Email: "tagashigeki@yahoo.co.jp
}

Received 22 May 2013; revised 20 June 2013; accepted 27 June 2013

Copyright (c) 2013 Shigeki Taga et al. This is an open access article distributed under the Creative Commons Attribution License, which permits unrestricted use, distribution, and reproduction in any medium, provided the original work is properly cited.

\begin{abstract}
Malignant spinal cord compression (MSCC) rarely presents in patients with endometrial cancer. It usually occurs months or years after the diagnosis of the primary tumor. A 65-year-old woman presented with a huge uterine tumor suspecting leiomyosarcoma. She underwent total abdominal hysterectomy and bilateral salpingo-oophorectomy. Histopathological examination revealed mixed carcinoma (small cell carcinoma and endometrioid adenocarcinoma) of the endometrium. She presented with upper abdominal pain, back pain, cystoplegia and paraplegia of lower extremities postoperatively. Magnetic resonance imaging revealed bone metastasis with destruction of the eighth thoracic vertebral body as the sites of metastasis. Vertebrectomy and irradiation therapy were performed for the lesion. Although she received systemic chemotherapy consisting of paclitaxel and carboplatin, she died 4 months after hysterectomy.
\end{abstract}

Keywords: Malignant Spinal Cord Compression; Endometrial Cancer

\section{INTRODUCTION}

Malignant spinal cord compression (MSCC) is one of the most disabling complications of cancer metastasis. Patients with endometrial carcinoma rarely present spinal cord or cauda equina compression secondary to epidural metastasis. It is an oncological emergency requiring prompt diagnosis and treatment. However, the early signs and symptoms of compression can be overlooked. It usually occurs months or even years after the diagnosis of the primary disease. Here we report a case of endometrial cancer that presented MSCC because of thoracic vertebral bone metastasis and underwent vertebrectomy and radiotherapy.

\footnotetext{
"Corresponding author.
}

\section{CASE REPORT}

A 65-year old postmenopausal woman, gravida 3, para 3, presented with vaginal bleeding and lower abdominal tumor and visited a local clinic, and was referred to our department. Ultrasound scans revealed a large pelvic mass with a diameter of $15 \mathrm{~cm}$. Cytological test of uterine cervix was negative. That of the endometrium was not possible. Abdominal CT scans revealed a huge pelvic mass partially carcified (Figure 1). No lymphadenopathy or ascites was noticed. Magnetic resonance imaging (MRI) revealed a heterogeneous solid tumor (Figure 2). Chest CT showed multiple lung metastases that seemed as metastases of the pelvic tumor (Figure 3). Serum level of LDH was elevated to $1519 \mathrm{U} / \mathrm{l}$, whereas other tumor markers were within normal range. Uterine leiomyosarcoma was suspected. The patient was admitted and total abdominal hysterectomy and bilateral salpingo-oophorectomy were carried out (Figure 4). Histopathological studies revealed mixed carcinoma (small cell carcinoma and endometrioid adenocarcinoma) with cervical invasion and peritoneal dissemination (Figure 5). Four days after surgery, she presented with upper abdominal pain and back pain. And 8 days after surgery, she presented with cystoplegia and paraplegia of lower extremities. MRI revealed bone metastasis with destruction of the eighth thoracic vertebral body as the sites of metastasis (Figure 6). She was diagnosed as having MSCC. Following administration of dexamethasone (6.6 $\mathrm{mg}, 20 \mathrm{mg}$ and $13.2 \mathrm{mg}$, consecutively), she underwent vertebrectomy and posterior spinal fusion, and postoperative radiation therapy was performed to total of 20 Gray in 4 fractions. The histopathological studies revealed malignant cells. Although artifacts made detailed histopathological diagnosis difficult, we clinically diagnosed this lesion as metastasis from endometrial cancer. However, her symptoms were not improved. Although she received two courses of systemic chemotherapy consisting of paclitaxel and carboplatin $\left(180 \mathrm{mg} / \mathrm{m}^{2}\right.$ and 


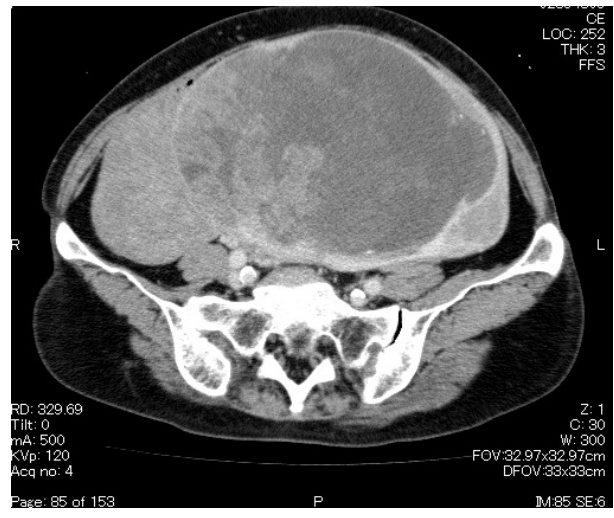

Figure 1. Abdominopelvic CT.

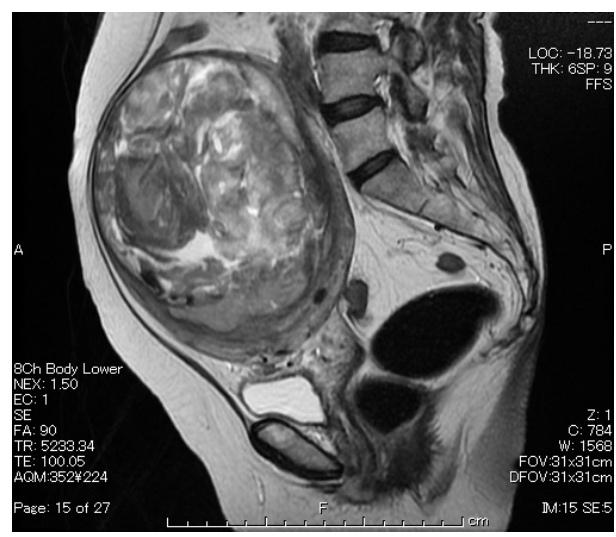

Figure 2. MRI of the pelvis.

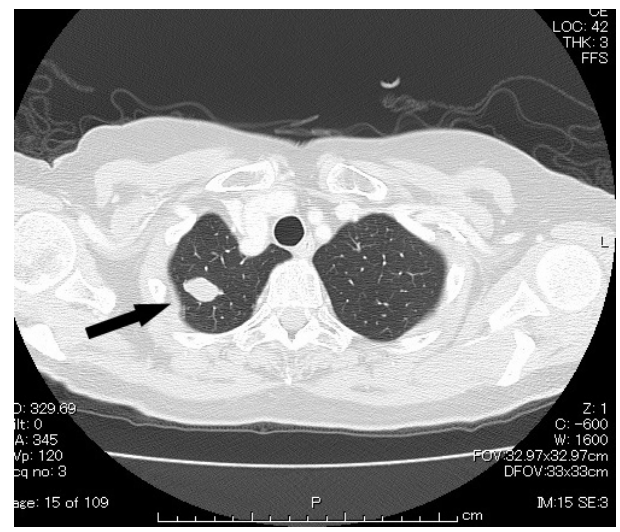

Figure 3. Chest CT revealed pulmonary metastases (arrow).

AUC $=6$, respectively), CT scan of the chest revealed new lung metastatic lesion, and she selected the best supportive care. She died 4 months after hysterectomy.

\section{DISCUSSION}

Although recent progress of chemotherapy has made the lives of cancer patients longer, the rate of bone metastasis is increasing accordingly. Bone is the third most frequent site of cancer metastasis next to lung and liver.

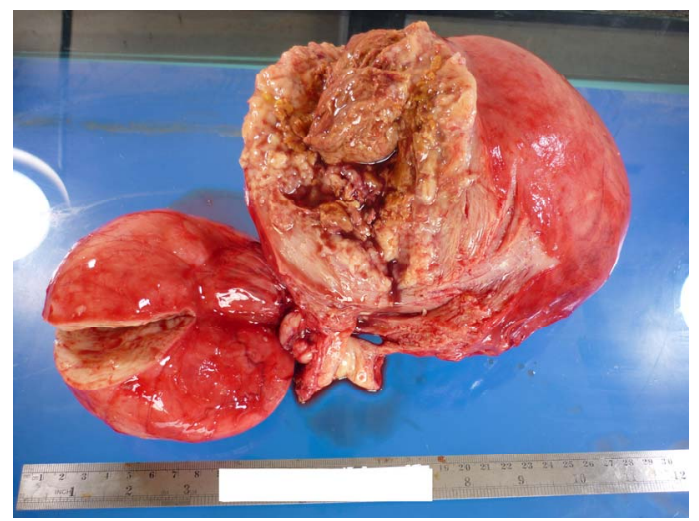

Figure 4. Surgical specimen.

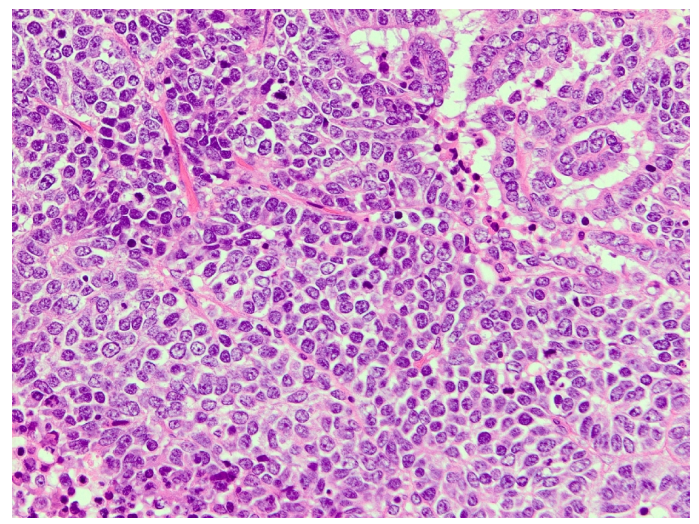

Figure 5. HES $\times$ 200: Histopathological studies revealed mixed carcinoma (small cell carcinoma and endometrioid adenocarcinoma).

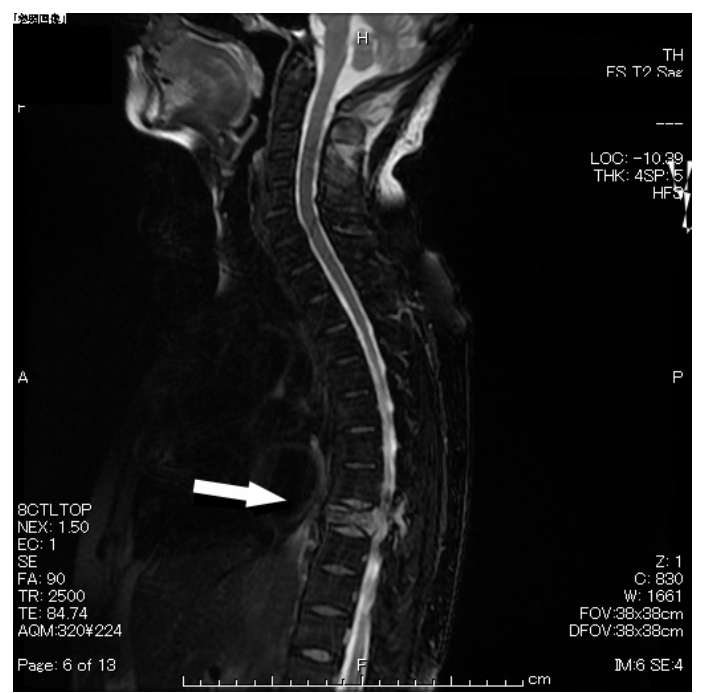

Figure 6. MRI of the spine revealed thoracic vertebral metastasis (arrow).

Breast cancer is the most common origin of bone metastasis, followed by lung cancer and uterine cancer. Approximately $70 \%$ of cancer patients have metastasis at their death [1]. The spine is the most common osseous 
site for metastatic disease and may be involved up to $40 \%$ of the patients with cancer, and $5 \%$ to $10 \%$ of all cancer patients may develop epidural spinal cord compression [2]. And $10 \%$ to $20 \%$ of patients with bony spinal disease develop symptomatic spinal cord compression [3]. The definition of MSCC is compression of the dural sac and its contents (spinal cord and/or cauda equine) by extradural tumor mass [4]. Endometrial cancer metastasizes to the bone less frequently than cervical cancer. Rome et al. stated in his review of 11 comprehensive series containing 1075 cases with all types of malignancy, that there were 35 cases of primary gynecologic malignancy and 3 cases were uterine corpus cancer. They reported that MSCC patients showed following symptoms: 1) back pain; 2) root pain; 3) motor disturbance; 4) sensory disturbance; 5) disturbance of bladder and/or bowel function [5]. Gilbert et al. reported that thoracic region was involved in $68 \%$ of metastatic extradural tumors, and pain was the primary symptom of $96 \%$ of the patients, while motor or sensory deficits were found in $82 \%$ of them [6]. The present case showed all those symptoms that Rome et al. stated and was diagnosed with MSCC. Although MSCC usually appear several months or years after the diagnosis of the primary disease, this patient presented it very early, as 1 week after operation.

As for diagnosis, Loblaw et al. reported that the sensitivity and specificity for MRI range from 0.44 to 0.93 and 0.90 to 0.98 , respectively, and that the sensitivity and specificity for myelography range from 0.71 to 0.97 and 0.88 to 1.00 , respectively. They concluded that MRI is the preferred imaging technique [4].

The management of MSCC consists of surgery, radiation and chemotherapy. And good evidence supports the use of steroids in patients with newly diagnosed MSCC. Dexamethasone is the most widely used steroid, although the optimal dose is controversial [7]. Radiotherapy has been the primary therapy for metastatic spinal disease. Until the mid-1980s, posterior decompressive laminectomy was regarded as the only surgical option, but its efficacy had been disappointing. However, surgery that decompresses the spinal cord circumferentially, followed by reconstruction and immediate stabilization has proven effective. Klimo et al. [8] reviewed 24 surgical articles (999 patients) and 4 radiation articles (543 patients). Overall ambulatory success rates for surgery and radiation were $85 \%$ and $64 \%$ respectively, and the cumulative rescue rates for the surgical and radiation literature were 0.58 and 0.26 respectively. They concluded that all patients diagnosed with metastatic spinal disease should be evaluated for surgery as a primary treatment modality. However, once neurological symptoms appear, they usually progress promptly, and the quality of life of the patient is harmed seriously. Currently, the results of treatment of neurological compression due to gynecologic malignancies are still unsatisfactory. As prevention is important, early diagnosis and good treatment of the primary disease are mandatory.

\section{REFERENCES}

[1] Bohm, P. and Huber, J. (2002) The surgical treatment of bony metastases of the spine and limbs. Journal of Bone \& Joint Surgery $(\mathrm{Br}), \mathbf{8 4}, 521-529$. doi:10.1302/0301-620X.84B4.12495

[2] Klimo Jr., P., Kestle, J.R.W. and Schmidt, M.H. (2004) Clinical trials and evidence-based medicine for metastatic spine disease. Neurosurgery Clinics of North America, 15, 549-564. doi:10.1016/j.nec.2004.04.016

[3] Klimo Jr., P. and Schmidt, M.H. (2004) Surgical management of spinal metastasis. The Oncologist, 9, 188-196. doi:10.1634/theoncologist.9-2-188

[4] Loblaw, D.A., Perry, J., Chambers, A. and Laperriere, N.J. (2005) Systematic review of the diagnosis and management of malignant extradural spinal cord compression: The Cancer Care Ontario Practice Guidelines Initiative's Neuro-Oncology Disease Site Group. Journal of Clinical Oncology, 23, 2028-2037. doi:10.1200/JCO.2005.00.067

[5] Rome, R.T. and Nelson Jr., J.H. (1977) Compression of the spinal cord or cauda equine complicating gynecological malignancy reports of five cases and a review of the literature. Gynecologic Oncology, 5, 273-290. doi:10.1016/0090-8258(77)90036-1

[6] Gilbert, R.W., Kim, J.H. and Posner, J.B. (1978) Epidural spinal cord compression from metastatic tumor: Diagnosis and treatment. Annals of Neurology, 3, 40-51. doi:10.1002/ana.410030107

[7] Schmidt, M.H., Klimo Jr., P. and Vrionis, F.D. (2005) Metastatic spinal cord compression. Journal of the $\mathrm{Na}$ tional Comprehensive Cancer Network, 3, 711-719.

[8] Klimo Jr., P., Thompson, C.J., Kestle, J.R. and Schmidt, M.H. (2005) A meta-analysis of surgery versus conventional radiotherapy for the treatment of metastatic spinal epidural disease. Neuro-Oncology, 7, 64-76. doi: $10.1215 /$ S1152851704000262 